Portland State University

PDXScholar

Civil and Environmental Engineering Faculty

Publications and Presentations

$12-1-2011$

\title{
Bottom Topography Mapping via Nonlinear Data Assimilation
}

\author{
Edward D. Zaron \\ Portland State University, ezaron@pdx.edu \\ Marie-Aude Pradal \\ Stevens Institute of Technology \\ Patrick D. Miller \\ Stevens Institute of Technology \\ Alan F. Blumberg \\ Stevens Institute of Technology \\ Nickitas Georgas \\ Stevens Institute of Technology
}

See next page for additional authors

Follow this and additional works at: https://pdxscholar.library.pdx.edu/cengin_fac

Part of the Civil and Environmental Engineering Commons

Let us know how access to this document benefits you.

\section{Citation Details}

Zaron, E. D., Pradal, M., Miller, P. D., Blumberg, A. F., Georgas, N., Li, W., \& Cornuelle, J. (2011). Bottom Topography Mapping via Nonlinear Data Assimilation. Journal of Atmospheric \& Oceanic Technology, 28(12), 1606-1623.

This Article is brought to you for free and open access. It has been accepted for inclusion in Civil and Environmental Engineering Faculty Publications and Presentations by an authorized administrator of PDXScholar. Please contact us if we can make this document more accessible: pdxscholar@pdx.edu. 


\section{Authors}

Edward D. Zaron, Marie-Aude Pradal, Patrick D. Miller, Alan F. Blumberg, Nickitas Georgas, Wei Li, and Julia Muccino Cornuelle 


\title{
Bottom Topography Mapping via Nonlinear Data Assimilation
}

\author{
EDWARD D. ZARON \\ Department of Civil and Environmental Engineering, Portland State University, Portland, Oregon \\ Marie-Aude Pradal, Patrick D. Miller, Alan F. Blumberg, Nickitas Georgas, \\ Wei Li, AND Julia MucCino CORNUElle \\ Center for Maritime Systems, Stevens Institute of Technology, Hoboken, New Jersey
}

(Manuscript received and in final form 18 April 2011)

\begin{abstract}
A variational data assimilation method is described for bottom topography mapping in rivers and estuaries using remotely sensed observations of water surface currents. The velocity field and bottom topography are related by the vertically integrated momentum and continuity equations, leading to a nonlinear inverse problem for bottom topography, which is solved using a Picard iteration strategy combined with a nonlinear line search. An illustration of the method is shown for Haverstraw Bay, in the Hudson River, where the known bottom topography is well reconstructed. Once the topography has been estimated, currents and water levels may be forecast. The method makes feasible 1) the estimation of bottom topography in regions where in situ data collection may be impossible, dangerous, or expensive, and 2) the calibration of barotropic shallow-water models via control of the bottom topography.
\end{abstract}

\section{Introduction}

Knowledge of bottom topography or water depth is of practical importance to marine navigation and other activities within rivers and estuaries, and is a crucial component of all realistic hydrodynamic models as well. In geophysical flows the role of bottom topography is significant at nearly all length scales, ranging from the topographic beta effect on large scales to boundary layer and kinematic effects on small scales. For example, in the earliest work with large-scale diagnostic ocean models a great sensitivity to the alignment of the bottom slope with respect to the vertically integrated baroclinic pressure gradient was found, the so-called Joint Effect of Baroclinicity and Relief (JEBAR) term (e.g., Sarkisyan and Ivanov 1971). Bottom topography also strongly influences the generation and propagation of both surface and internal gravity waves. These effects are of demonstrable practical importance in storm surge modeling (Heemink et al. 2002), regional ocean modeling (Hirose

Corresponding author address: Edward D. Zaron, P.O. Box 751, Department of Civil and Environmental Engineering, Portland State University, Portland, OR 97207-0751.

E-mail: zaron@cecs.pdx.edu
2005), and coupled ocean-estuary modeling (Blumberg and Georgas 2008).

It is precisely the wide range of significant length scales that makes the determination of topography by direct methods (e.g., depth-finding sonar) a challenging and costly endeavor, and provides the impetus for the present work. Our focus here is on the determination of bottom depth using remotely sensed observations of currents at the water surface, such as may be obtained from a variety of measurement systems. The basic principle of these systems is to measure the Doppler shift of the surface waves induced by the horizontal component of the near-surface water velocity. The representative site considered here is Haverstraw Bay, within the Hudson River estuary system, where surface current data have been obtained using the Airborne Remote Optical Spotlight System (AROSS; Dugan et al. 2001a,b). Emphasis is on the use of surface currents to map bottom topography on horizontal scales of $100 \mathrm{~m}$ or more, over a $10 \mathrm{~km} \times$ $50 \mathrm{~km}$ area.

Previous work has focused on using water level data to calibrate hydrodynamic model parameters, including bottom topography (e.g., Das and Lardner 1991; Lardner et al. 1993; Ten-Brummelhuis et al. 1993; Heemink et al. 2002). For example, Heemink et al. (2002) utilize observations of 
water level to adjust values of bottom depth, roughness coefficient, vertical viscosity, and open boundary conditions. Their formulation utilizes the adjoint of a hydrodynamic model to efficiently calculate the gradient of an objective function, consisting of the sum-of-squared residuals of observed and predicted water levels. Control variables are treated as spatially constant within discrete subdomains; hence, calibrated values of bottom depth, etc., are spatially discontinuous using their approach.

Losch and Wunsch (2003) conduct a theoretical study to identify large-scale bottom topography from sea surface height data using adjoint-based methods. Constraints on spatial regularity (smoothness) are found necessary when the surface observations contain realistic levels of error. The importance of a priori information, the firstguess topography, is also emphasized because the surface data do not uniquely determine the bottom topography where there are regions of weak flow or when the data are noisy.

Mourre et al. (2004) consider the estimation of bottom topography from water elevation measurements in the context of tidal modeling. A statistical model for the spatial distribution of errors is hypothesized, and an ensemble of plausible bottom topography fields is created in order to determine the cross correlation between water elevation and bottom depth. Statistical linearization is invoked to estimate bottom topography consistent with observed water levels.

Hirose (2005) utilizes observations from a ship-mounted acoustic Doppler current profiler (ADCP) to calibrate bottom topography in a coastal ocean model. The approach is somewhat different from those described previously in that the linear combination of four different gridded bathymetry datasets is found, which minimizes the misfit between the observed and modeled currents.

The estimation of topography from surface currents in this paper follows the adjoint-based approaches just discussed, but with some differences in solver structure and implementation. For example, the minimization algorithm used here consists of an inner linear solver, based on an expansion in terms of representer functions (Bennett 1992, 2002), and an outer series of functional, or Picard, iterations that decouple the nonlinearity from the inner solver. This approach permits flexibility in the number and quality of data to be assimilated; thus, it is not necessary to provide observations at every model grid point or to reduce the number of topography parameters to be estimated, as has been done previously. The solver architecture naturally lends itself to an analysis of the conditioning, sensitivity, and properties of the observational array, and posterior errors may be estimated.
The outline of this paper is as follows. In section 2 the topography estimation problem is defined, the solution algorithm is outlined, and an idealized example is presented to illustrate the performance of the solver. In section 3 the central scientific issue is addressed, namely, how to assign reasonable bounds to the accuracy and spatial covariance of the prior topography. A proof-of-concept demonstration is described for an estuary with known topography in section 4. Finally, sections 5 and 6 discuss important remaining questions and summarize our results.

\section{Formulation and implementation}

There are two features of the topography estimation problem that make it a challenge. First, the coupling between bottom depth and water velocity is strongly nonlinear, arising from both kinematics (mass continuity) and dynamics (vertically integrated pressure gradient), as already mentioned. Second, the unknown bottom topography is a continuous field; in order to estimate it from a finite number of observations it is necessary to hypothesize a model for statistics of the topography, minimally, its mean and (spatial) autocovariance function.

An estimator for the bottom topography is proposed that is the minimizer of a quadratic form, or objective function, which is the weighted sum of squared misfits between a set of predicted and observed currents, an integral over the deviation of the topography from a first guess, and an integral over dynamical error. Although the objective function is quadratic in the above-mentioned error terms, it is a nonquadratic (and nonconvex) function of the bottom depth because of the nonlinear coupling between current and bottom depth in both the momentum and continuity equations. The strategy for handling the nonlinearity is based on functional iteration using a sequence of linear inverse problems solved by standard methods of variational data assimilation.

\section{a. Problem formulation}

It is assumed that the dominant influence of the bottom topography on the currents is well represented by barotropic shallow-water dynamics. Thus, baroclinic currents caused by variations in water density are neglected, and the pressure is assumed to be in hydrostatic balance, consistent with the small aspect ratio of the flow. Typical applications are weakly stratified bays or estuaries, with water depth ranging from a fraction of a meter to some tens of meters and topography variations on scales from hundreds of meters to kilometers.

In practice, the hydrodynamic model is solved in general orthogonal curvilinear coordinates, but for simplicity 
Cartesian coordinates are used below, with $x$ and $y$ denoting the zonal and meridional coordinates, respectively, and $\mathcal{D}$ denoting the spatial domain. The time dimension is denoted as $t \in[0, T]$, so that $(x, y, t) \in \mathcal{D} \times[0, T]$. It is assumed that the boundary of the domain $\partial \mathcal{D}$ is composed of closed and open boundary segments, $\partial \mathcal{D}_{1}$ and $\partial \mathcal{D}_{2}$, respectively. Within $\mathcal{D}$ the dependent variables are water elevation (referenced to equilibrium) $\eta$, zonal velocity $u$, and meridional velocity $v$. Bottom topography, the undisturbed water depth, is denoted as $H$; it is assumed to be constant in time; and the total water depth at any instant is $D=H+\eta$.

Following the general approach of weak-constraint variational data assimilation, it is assumed that all of the governing equations, including boundary and initial conditions, contain error. The errors are denoted by $\hat{\lambda}^{*}$, with the superscript $*$ indicating within which equation the error appears. Allowing for these errors, the barotropic shallow-water equations are

$$
\begin{gathered}
\eta_{t}+(u D)_{x}+(v D)_{y}=\hat{\lambda}^{\eta} \\
(D u)_{t}+\left(D u u-2 D A_{m} u_{x}\right)_{x}+\left[D v u-D A_{m}\left(u_{y}+v_{x}\right)\right]_{y} \\
-f D v+g D \eta_{x}+C_{d}\left(u^{2}+v^{2}\right)^{1 / 2} u=\hat{\lambda}^{u} \\
(D v)_{t}+\left[D u v-D A_{m}\left(u_{y}+v_{x}\right)\right]_{x}+\left(D v v-2 D A_{m} v_{y}\right)_{y} \\
+f D u+g D \eta_{y}+C_{d}\left(u^{2}+v^{2}\right)^{1 / 2} v=\hat{\lambda}^{v} \\
D=H+\eta+\hat{\lambda}^{D} \\
H=H_{0}+\hat{\lambda}^{H}
\end{gathered}
$$

where $H_{0}$ is an initial guess of the topography, $A_{m}$ is the horizontal eddy viscosity, $C_{d}$ is the bottom drag coefficient, $f$ is the Coriolis parameter, and $g$ is the acceleration of gravity. Note that, in the numerical implementation, $A_{m}$ is a nonlinear function of the local strain rate via the Smagorinsky-Lilly scheme (Smagorinsky 1963; Lilly 1967), $C_{d}$ is spatially constant, and $f$ is a function of geographic latitude.

Boundary conditions are approximately no slip on closed (material) boundaries,

$$
(u, v)=\left(\hat{\lambda}^{\partial D_{1} u}, \hat{\lambda}^{\partial D_{1} v}\right) \quad \text { on } \quad \partial \mathcal{D}_{1} .
$$

On open boundaries the surface elevation $\eta_{d}$ is specified as

$$
\eta=\eta_{d}+\hat{\lambda}^{\partial D_{2}} \quad \text { on } \quad \partial \mathcal{D}_{2},
$$

and no stress is assumed for the horizontal viscous terms. Initial conditions, which may also contain errors, are given by $\left(u_{0}, v_{0}, \eta_{0}\right)$ for the horizontal velocity components and surface elevation

$$
(u, v, \eta)=\left(u_{0}, v_{0}, \eta_{0}\right)+\left(\hat{\lambda}^{u_{0}}, \hat{\lambda}^{v_{0}}, \hat{\lambda}^{\eta_{0}}\right)
$$

As mentioned above, the unknown corrections to the dynamics, initial, and boundary conditions are denoted by the $\hat{\lambda}^{*}$ terms. The hypothesized second-order statistics (covariances) of these unknowns are denoted $C^{*}$, where the superscript $*$ indicates the corresponding field. The bottom topography will be found, which minimizes a weighted sum of squares of the error terms, with the weights being the inverse of the respective covariances.

In addition to the dynamical information, specified above, there are observational data consisting of a set of $M$ discrete measurements of surface currents. Each measurement is represented as the projection of the velocity field onto a measurement kernel $\left(\mu_{i}, \nu_{i}\right)$, plus an unknown measurement error $\epsilon_{i}$,

$$
u_{i}=\int_{0}^{T} d t \int_{\mathcal{D}} d x d y\left(\mu_{i}, \nu_{i}\right) \cdot(u, v)+\epsilon_{i}
$$

for $i=1, \ldots, M$. Measurement operators $\left(\mu_{i}, \nu_{i}\right)$ here consist of averaging kernels defined by the footprint of the remote sensing system. As noted in the introduction, the measurements are obtained by a Doppler technique that measures orthogonal components of the surface current over spatial patches of approximately $128 \mathrm{~m} \times$ $128 \mathrm{~m}$, with an averaging time of approximately $30 \mathrm{~s}$ (Dugan et al. 2001b; Dugan and Piotrowski 2003). Other measurements may be assimilated, for example, measurements of water elevation $\eta$ or in situ measurements of $H$, and these are included in the implementation described below.

The topographic estimation problem is posed by seeking fields $(u, v, \eta, H)$, which minimize the weighted sum of squared errors in the dynamics and the data, denoted $\mathcal{J}$. When the errors are normally distributed with known covariance, the minimizer of $\mathcal{J}$ is a maximum likelihood estimator (Bennett 2002). Knowledge of the bottom topography and dynamical error statistics is extremely limited in the present case, so the least squares formulation is regarded as a useful expedient for finding a regularized estimate of $H$, consistent with both the dynamics and the data. Although the $C^{*}$ operators will be referred to as "covariances," they are constructed from rough estimates of the magnitude and correlation scales of the corresponding terms.

The objective function $\mathcal{J}$ to be minimized is 


$$
\begin{aligned}
\mathcal{J}(u, v, \eta, H)= & \int_{0}^{T} d t \int_{\mathcal{D}} d x d y\left[\eta_{t}+(u D)_{x}+(v D)_{y}\right] \lambda^{\eta} \\
& +\int_{0}^{T} d t \int_{\mathcal{D}} d x d y\left\{(D u)_{t}+\left(D u u-2 D A_{m} u_{x}\right)_{x}+\left[D v u-D A_{m}\left(u_{y}+v_{x}\right)\right]_{y}-f D v+g D \eta_{x}\right. \\
& \left.+C_{d}\left(u^{2}+v^{2}\right)^{1 / 2} u\right\} \lambda^{u} \\
& +\int_{0}^{T} d t \int_{\mathcal{D}} d x d y\left\{(D v)_{t}+D u v-D A_{m}\left(u_{y}+v_{x}\right)_{x}+\left(D v v-2 D A_{m} v_{y}\right)_{y}+f D u+g D \eta_{y}\right. \\
& \left.+C_{d}\left(u^{2}+v^{2}\right)^{1 / 2} v\right\} \lambda^{v} \\
& +\int_{0}^{T} d t \int_{\mathcal{D}} d x d y(D-H-\eta) \lambda^{D} \\
& +\int_{\mathcal{D}}^{T} d x d y\left(H-H_{0}\right) \lambda^{H} \\
& +\int_{0}^{T} d t \int_{\partial D_{1}} d l(u, v) \cdot \mathbf{n} \lambda^{\partial D_{1}} \\
& +\int_{0}^{T} d t \int_{\partial D_{2}} d l\left(\eta-\eta_{d}\right) \lambda^{\partial D_{2}} \\
& +\int_{\mathcal{D}}^{M} d x d y\left[(u, v, \eta)-\left(u_{0}, v_{0}, \eta_{0}\right)\right] \cdot\left(\lambda^{u 0}, \lambda^{v 0}, \lambda^{\eta 0}\right) \\
& +\sum_{i=1 v}^{M}\left[\int_{0}^{T} d t \int_{\mathcal{D}} d x d y\left(\mu_{i}, \nu_{i}\right) \cdot(u, v)-u_{i}\right]^{2} / \sigma_{i}^{2}
\end{aligned}
$$

where the adjoint variables $\lambda^{*}$ and the fields $\hat{\lambda}^{*}$ in (1)(10) are related by Bennett (1992),

$$
\hat{\lambda}^{*}=C^{*}{ }^{*} \lambda^{*}
$$

hence, the terms in the sum (12) are implicitly weighted by the corresponding $\left(C^{*}\right)^{-1}$. Note that each $C^{*}$ is a function of two sets of space-time arguments, and the inner product $\circ *$ depends on the domain on which $\hat{\lambda}^{*}$ is defined. For example, on $\partial D_{2}$, let $l$ parameterize the distance along the open boundary $(l, t) \in \partial D_{2} \times[0, T]$; then, the inner product $o^{\partial \mathcal{D}_{2}}$ is defined as

$$
\begin{aligned}
\hat{\lambda}^{\partial D_{2}(l, t)} & =C^{\partial D_{2} \partial \partial D_{2}} \lambda^{\partial D_{2}} \\
& =\int_{0}^{T} d t^{\prime} \int_{\partial D_{2}} d l^{\prime} C^{\partial D_{2}}\left(l, t ; l^{\prime}, t^{\prime}\right) \lambda^{\partial D_{2}}\left(l^{\prime}, t^{\prime}\right) .
\end{aligned}
$$

For simplicity, it has been assumed that the observation errors are uncorrelated, and the standard deviation of the $i$ th measurement is $\sigma_{i}$. Likewise, the separate components of the model errors are assumed to be uncorrelated, for example, the expected value of $\lambda^{*} \lambda^{\star}$ is zero for components $* \star \star$.

The first-order extremal conditions for the objective function are found by setting to zero the variation with respect to each of the dependent variables. Nonlinear
Euler-Lagrange equations (E-L) are obtained, which express the first-order optimality conditions for $\mathcal{J}$. Direct solution of the E-L equations for the optimal bottom topography is difficult, although the discretized equations may be solved by quasi-Newton methods when relatively few values of $H$ are to be estimated (Heemink et al. 2002; Losch and Wunsch 2003). In the next section we derive a solution algorithm that utilizes the intrinsic number of degrees of freedom in the estimation problem, namely, the number of $(u, v)$ measurements $M$, to obtain a topography solver whose conditioning is essentially independent of the number of grid unknowns.

\section{b. Solution algorithm}

The key practical difficulty with solving the E-L system is the nonlinear coupling between $H$ and the other variables. If the system were linear, the E-L system could be reduced to an $M$-dimensional linear system using the wellknown duality between the state-space and observationspace representations of variational data assimilation (Bennett 1992; Courtier 1997). We exploit this duality by approximating the solution of the nonlinear E-L system with the limit of a sequence of linear E-L systems constructed using Picard iteration.

There are no general proofs of convergence for Picard iteration applied to nonlinear E-L systems, but three 
basic principles were utilized in developing a solver. First, each linear approximation to the nonlinear E-L system should itself be an E-L system, representing the extremal conditions for a quadratic (strictly convex) objective function. Second, the corresponding linear dynamics should be chosen in order to possess a bounded energy-like integral, which simplifies the development of stable and accurate numerics for the E-L equations. And, third, rather than taking the solution of the linear $\mathrm{E}-\mathrm{L}$ system as an approximation to the minimum of $\mathcal{J}$ directly, it is used to define the search direction for a linesearch optimization. The first two of these principles were suggested by Bennett (2002) for nonlinear data assimilation. The third principle was adopted in order to accommodate the errors caused by linearization; it ensures that the linearized solver does at least yield a nonincreasing sequence of $\mathcal{J}$.

After a systematic search of linear approximations possessing energy-like integrals, we found the following system could be integrated stably:

$$
\begin{aligned}
& \eta_{t}^{n+1}+\left(u^{n+1} \bar{D}\right)_{x}+\left(v^{n+1} \bar{D}\right)_{y}+\left[\bar{u}\left(H^{n+1}-\bar{H}\right)\right]_{x} \\
& \quad+\left[\bar{v}\left(H^{n+1}-\bar{H}\right)\right]_{y}=\hat{\lambda}^{\eta}
\end{aligned}
$$

$$
\begin{aligned}
& \left(\bar{D} u^{n+1}\right)_{t}+\left(\bar{D} \bar{u} u-2 \bar{D} A_{m} \bar{u}_{x}\right)_{x}+\left[\bar{D} \bar{v} \bar{u}-\bar{D} A_{m}\left(\bar{u}_{y}+\bar{v}_{x}\right)\right]_{y} \\
& \quad-f \bar{D} \bar{v}+g \bar{D} \eta_{x}^{n+1}+g\left(H^{n+1}-\bar{H}\right) \bar{\eta}_{x} \\
& \quad+C_{d}\left(\bar{u}^{2}+\bar{v}^{2}\right)^{1 / 2} u^{n+1}=\hat{\lambda}^{u}
\end{aligned}
$$

$$
\begin{gathered}
\left(\bar{D} v^{n+1}\right)_{t}+\left[\bar{D} \bar{u} \bar{v}-\bar{D} A_{m}\left(\bar{u}_{y}+\bar{v}_{x}\right)\right]_{x}+\left(\bar{D} \bar{v} v-2 \bar{D} A_{m} \bar{v}_{y}\right)_{y} \\
+f \bar{D} \bar{u}+g \bar{D} \eta_{y}^{n+1}+g\left(H^{n+1}-\bar{H}\right) \bar{\eta}_{y} \\
+C_{d}\left(\bar{u}^{2}+\bar{v}^{2}\right)^{1 / 2} v^{n+1}=\hat{\lambda}^{v} \\
H^{n+1}=H_{0}+\hat{\lambda}^{H}
\end{gathered}
$$

where superscript $n$ denotes the Picard iteration count [which is omitted from both $\hat{\lambda} *$ and $(\bar{u}, \bar{v}, \bar{\eta}, \bar{H})$ terms for readability]. The overbar denotes the background field used in the linear approximation; it is the result of a line search from the previous iteration, for example,

$$
\begin{aligned}
\left(\bar{u}^{n+1}, \bar{v}^{n+1}, \bar{\eta}^{n+1}, \bar{H}^{n+1}\right)= & \left(\bar{u}^{n}, \bar{v}^{n}, \bar{\eta}^{n}, \bar{H}^{n}\right)\left(1-\alpha^{n}\right) \\
& +\left(u^{n+1}, v^{n+1}, \eta^{n+1}, H^{n+1}\right) \alpha^{n},
\end{aligned}
$$

where scalar $\alpha^{n}$ is chosen to minimize $\mathcal{J}$ considered as a function of $\alpha^{n}$ alone. Note that, consistent with previous approximations, the turbulent viscosity $A_{m}$ is a function of $\left(\bar{u}^{n}, \bar{v}^{n}\right)$ as well.

Boundary conditions for the linearized system are given by

$$
\begin{aligned}
& \left(u^{n+1}, v^{n+1}\right) \\
& =\left(\hat{\lambda}^{\partial D_{1} u}, \hat{\lambda}^{\partial D_{1} v}\right) \text { on } \partial D_{1} \text { (closed boundary), }
\end{aligned}
$$

and

$$
\eta^{n+1}=\eta_{d}+\hat{\lambda}^{\partial D_{2}} \text { on } \partial D_{2} \text { (open boundary). }
$$

Initial conditions are

$\left(u^{n+1}, v^{n+1}, \eta^{n+1}\right)=\left(u_{0}, v_{0}, \eta_{0}\right)+\left(\hat{\lambda}^{u 0}, \hat{\lambda}^{v 0}, \hat{\lambda}^{\eta 0}\right)$.

Finally, the first iteration for the background field $\left(\bar{u}^{1}, \bar{v}^{1}, \bar{\eta}^{1}, \bar{H}^{1}\right)$ is computed from (1) to (10) with $\hat{\lambda}^{*}=0$.

The so-called adjoint model, which consists of the E-L equations for the extremum of the quadratic objective function $\mathcal{J}_{n+1}$, is given by

$$
\begin{gathered}
-\lambda_{t}^{\eta}-\left(g \bar{D} \lambda^{u}\right)_{x}-\left(g \bar{D} \lambda^{v}\right)_{y}=0 \\
-\bar{D} \lambda_{t}^{u}-\bar{D} \lambda_{x}^{\eta}+C_{d}\left(\bar{u}^{2}+\bar{v}^{2}\right)^{1 / 2} \lambda^{u} \\
=-\sum_{i=1}^{M} \mu_{i} / \sigma_{i}^{2}\left[\int_{0}^{T} d t \int_{\mathcal{D}} d x d y\left(\mu_{i}, \nu_{i}\right) \cdot\left(u^{n+1}, v^{n+1}\right)-u_{i}\right]
\end{gathered}
$$

$$
\begin{aligned}
& -\bar{D} \lambda_{t}^{v}-\bar{D} \lambda_{y}^{\eta}+C_{d}\left(\bar{u}^{2}+\bar{v}^{2}\right)^{1 / 2} \lambda^{v} \\
& =-\sum_{i=1}^{M} \nu_{i} / \sigma_{i}^{2}\left[\int_{0}^{T} d t \int_{0} d x d y\left(\mu_{i}, \nu_{i}\right)\left(u^{n+1}, v^{n+1} v\right)-u_{i}\right],
\end{aligned}
$$

with a no-normal-flow-like boundary condition on $\partial D_{1}$,

$$
g \bar{D}\left(\lambda^{u}, \lambda^{v}\right) \cdot \mathbf{n}=0,
$$

where $\mathbf{n}$ is the outer normal, and a homogeneous elevationlike boundary condition on $\partial D_{2}$,

$$
\bar{D} \lambda^{\eta}=0 .
$$

At $t=T$, the final conditions are $\lambda^{\eta}=\lambda^{u}=\lambda^{v}=0$. Corrections to the bottom topography are computed by solving for $\lambda^{H}$,

$\lambda^{H}=-\int_{0}^{T} d t\left(-\bar{u} \lambda_{x}^{\eta}-\bar{v} \lambda_{y}^{\eta}+g \lambda^{u} \bar{\eta}_{x}+g \lambda^{v} \bar{\eta}_{y}\right)$

and using $\hat{\lambda}^{H}=C^{H}{ }^{\circ}{ }^{\mathcal{D}} \lambda^{H}$ to obtain $H^{n+1}$ in (18). Once the stopping criteria have been reached, the estimated topography will be denoted $H_{\text {est }}=\bar{H}^{n+1}$.

There are many implementation details. The forward and adjoint models are discretized following Blumberg and Mellor (1987) on an approximately orthogonal curvilinear 


\section{A) Outer-Loop Algorithm}

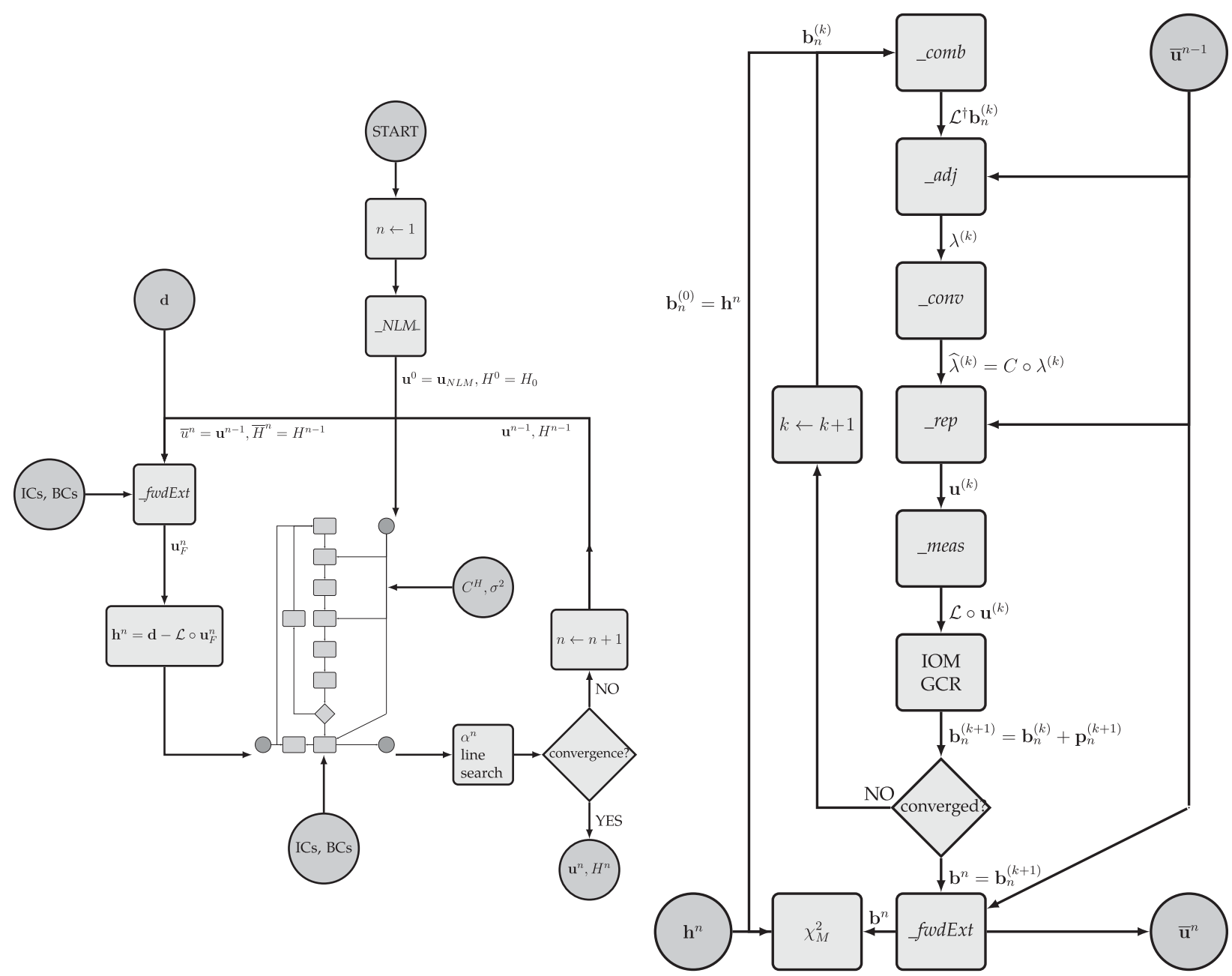

B) Inner-Loop Algorithm

FIG. 1. Solution Algorithm. Left: Schematic of nonlinear data assimilation algorithm. Beginning at the top, with the execution of the nonlinear model (_NLM), the Picard iteration proceeds counterclockwise. Measured data are denoted d, and "ICs, BCs" denote initial conditions and boundary conditions, respectively. The inner-loop, shown with unlabeled boxes, is enlarged on the right. Right: The indirect representer algorithm, the solver for the linear E-L system, (15)-(27), is shown. The outer Picard iteration index is denoted $n$, while the inner GCR iteration is denoted $k$. The leading underscore, _NLM,_fwdExt, etc., denotes software components using the notation of the IOM system (Bennett et al. 2008; Muccino et al. 2008).

grid. Nonlinear terms proportional to grid curvature (Blumberg and Herring 1987) are nonconservative when linearized and are omitted from the linear E-L system; however, they are retained in $\mathcal{J}$ and the nonlinear line search. Integration of the linearized system uses spaceand time-dependent background fields $(\bar{u}, \bar{v}, \bar{\eta})$; for computational efficiency these have been temporally subsampled and reconstructed as needed by linear interpolation. The solver for (15)-(27) is implemented with the indirect representer algorithm (Bennett 2002, section 3.1.5; Chua and Bennett 2001) using a generalized conjugate residual (GCR) solver. GCR, a Krylov subspace method applicable to nonsymmetric linear systems (de
Sturler 1994), was chosen because the implementation of Eqs. (23)-(27) is not the discrete adjoint of (15)-(22). Brent's method (Brent 1973) is the line search algorithm for $\alpha_{n}$. In practice, an acceptable topography estimate is usually obtained after two to four iterates. The computational effort necessary is equivalent to $40-120$ model integrations, depending on the geometry of the domain, the assumed level of error in the first-guess topography and surface current data, and the correlation scales of the actual and assumed errors.

Figure 1 shows a functional schematic of the solution algorithm and software components. The topography solver was implemented as an extension to the Inverse 
TABLE 1. Representative parameters for experiments.

\begin{tabular}{lll}
\hline \multicolumn{1}{c}{ Parameter } & Symbol & Value or range \\
\hline Cross-channel resolution & $\Delta x$ & $0.1-0.2 \mathrm{~km}$ \\
Along-channel resolution & $\Delta y$ & $0.2-4 \mathrm{~km}$ \\
Time step & $\Delta t$ & $5 \mathrm{~s}$ \\
Integration time & $T$ & $36 \mathrm{~h}$ \\
Bottom drag coefficient & $C_{d}$ & $2.5 \times 10^{-3}$ \\
Subsampling interval & & $300 \mathrm{~s}$ \\
$u$ measurement error & $\sigma$ & $0.1-0.2 \mathrm{~m} \mathrm{~s}^{-1}$ \\
Initial guess topography & $H_{0}$ & $5-15 \mathrm{~m}$ \\
Topography error magnitude & $\Delta H$ & $2-20 \mathrm{~m}, 0.25 \bar{H}$ \\
Topography error correlation length & $L_{x}, L_{y}$ & $0.2-15 \mathrm{~km}$ \\
Inner loop convergence criterion & & $0.02-0.2$ \\
Line search convergence criterion & & $0.1-0.25$ \\
Maximum Picard iterations & & $5-10$ \\
Wall-clock model run time & & $3-30 \mathrm{~min}$ \\
Wall-clock $H$ estimation & & $1-24 \mathrm{~h}$ \\
\hline
\end{tabular}

Ocean Model (IOM; see Bennett et al. 2008; Muccino et al. 2008), a software toolkit for developing variational data assimilation methods.

To reduce the impact of the first-guess topography $H_{0}$, a modification of the above algorithm has been implemented. The algorithm has been modified to make $H_{0}$ a function of the Picard iteration number $H_{0} \rightarrow H_{0}^{n}$, where $H_{0}^{n}$ is set equal to the optimized topography obtained from the previous Picard iteration $H_{0}^{n}=\bar{H}^{n}$. This change in the algorithm makes the solver analogous to the method of successive corrections (Cressman 1959), but it breaks the relationship to conventional least squares estimation. Although we have no formal justification for this procedure, we have found that it works well in practice.

Table 1 lists the range of parameter values used in the numerical model and topography solver.

\section{c. Solver validation}

To validate the solver described above, a series of experiments were conducted to verify that the true topography could be reconstructed within an idealized domain. These experiments provided the opportunity to validate the forward and adjoint solvers, profile computational performance, and test a variety of linearization strategies. Furthermore, identical twin experiments were conducted with a straight channel and a curved channel to analyze the impact of measurement noise, domain geometry, and other factors on the accuracy of the solutions.

As a basic demonstration, consider the problem of identifying the bottom topography in a 10-km-wide curved channel with tidal forcing provided at one end. The geometry of the channel is a half-annulus, the curvilinear grid is aligned with the radial and azimuthal directions, and the true topography consists of a "navigational channel," which obliquely crosses the annulus (see Fig. 2). In this and subsequent examples, all model, initial condition, and boundary errors are zero, $\hat{\lambda}^{*}=0$, except for $\hat{\lambda}^{H}$, which is the correction to the first-guess bottom topography. ${ }^{1}$ The first-guess depth is $H_{0}=8 \mathrm{~m}$, and $C^{H}$, the spatial autocovariance function of $H_{0}$, is a bell-shaped (Gaussian) function with principle axes aligned with the curvilinear coordinates,

$$
\begin{aligned}
C^{H}\left(x_{1}, y_{1}, x_{2}, y_{2}\right)= & (\Delta H)^{2} \exp \\
& \times\left[-\frac{1}{2}\left(\frac{\left(x_{1}-x_{2}\right)^{2}}{L_{x}^{2}}+\frac{\left(y_{1}-y_{2}\right)^{2}}{L_{y}^{2}}\right)\right],
\end{aligned}
$$

where $x_{i}$ and $y_{i}$ are across- (radial) and along- (azimuthal) channel coordinates. Correlation scales are $L_{x}=1.8 \mathrm{~km}$ and $L_{y}=14.0 \mathrm{~km}$, and the amplitude is scaled with $\Delta H=$ $5 \mathrm{~m}$. Measurement error is simulated with Gaussian noise $\sigma=0.5 \mathrm{~cm} \mathrm{~s}^{-1}$ added to the true currents.

Figure 2 shows the reconstructed bottom depth when water data are assimilated at a single time within the swath indicated, and the measured currents are being observed from an identical twin model containing the known topography. The depth field is well reconstructed within and nearby the swath of observations. The reconstruction loses accuracy away from the observations, especially at the closed end of the annulus, where currents are lower. An examination of Eq. (28) indicates that corrections to the bottom depth require that either of the terms $\left(\bar{u} \lambda_{x}^{\eta}+\bar{v} \lambda_{y}^{\eta}\right)$ or $\left(\lambda^{u} \bar{\eta}_{x}+\lambda^{v} \bar{\eta}_{y}\right)$ are nonzero (provided the terms do not cancel). Hence, topographic adjustments are generally largest where either currents $(\bar{u}, \bar{v})$ or the surface pressure gradient $(\nabla \bar{\eta})$ is large. Near the closed end of the channel (and far from the data) all of the terms are small, and the first-guess topography is not corrected. Closer to the measurement sites, the measurement kernels provide impulses to the $\left(\lambda^{u}, \lambda^{v}\right)$ equations, resulting in nonzero $\lambda^{\eta}$ via the adjoint continuity Eq. (23). Because significant currents are present, the terms in Eq. (28) are large, yielding corrections to $H$.

This example demonstrates in an idealized setting that it is possible to obtain useful information about the bottom depth, for example, the approximate location and depth of a navigational channel, using a very poor first guess, a relatively small amount of water current data, and no in situ data except for the water line (i.e., the lateral boundary of the domain) and elevation at the open boundary.

In practice, the key determinants of the accuracy of the estimated topography are the quality of the first

\footnotetext{
${ }^{1}$ Because $C^{H}$ is the sole nonzero error covariance function, $\hat{\lambda}^{H}$ is the only nonzero inhomogeneity to appear on the right-hand side of (1)-(10). The other $\lambda *$ terms are Lagrange multipliers in this case.
} 

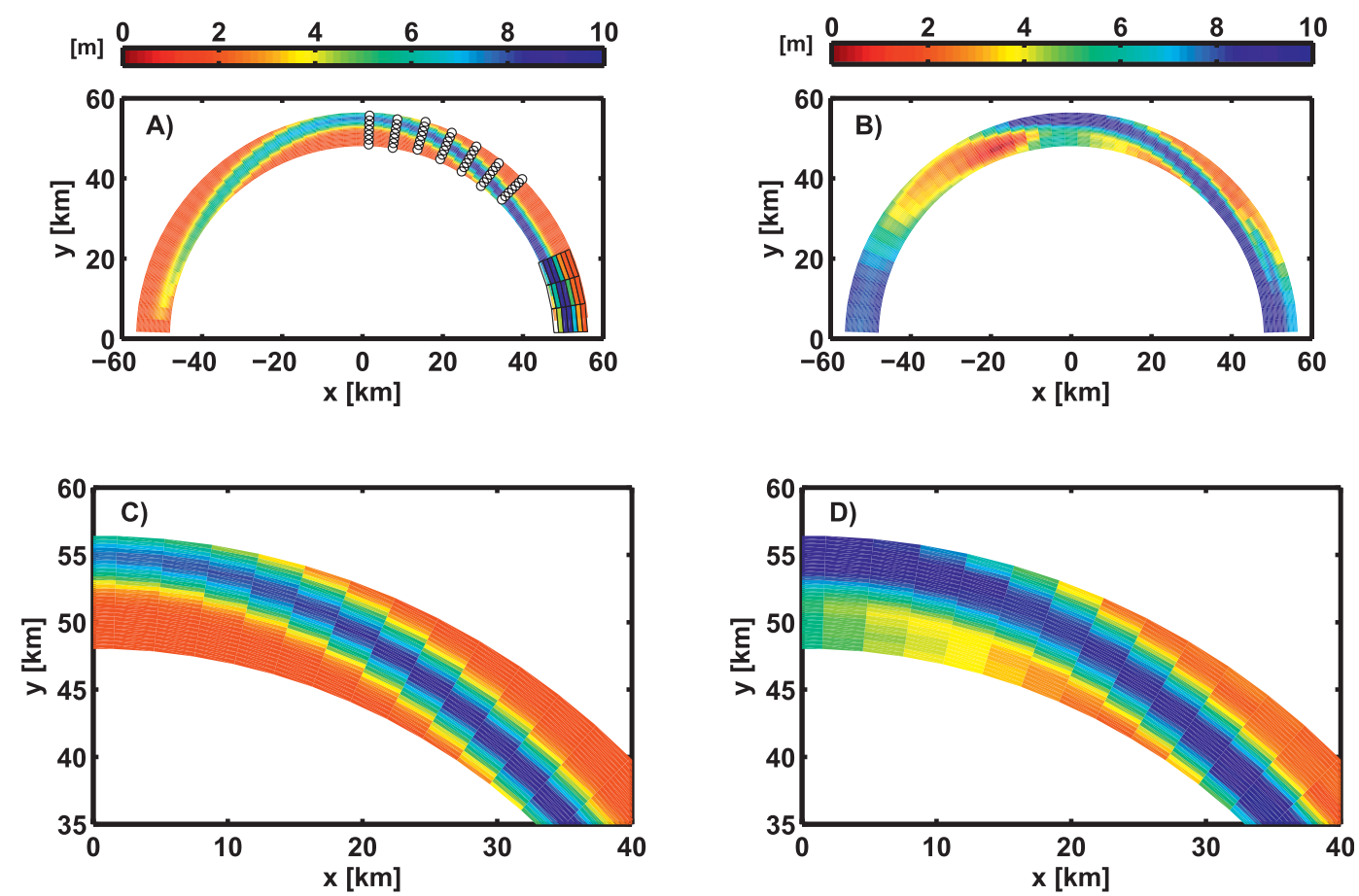

FIG. 2. Idealized curved channel. Software validation was performed with experiments in an idealized curved channel, where flow is driven by tidal forcing at the open boundary (near $x=55 \mathrm{~km}, y=0 \mathrm{~km}$ ). (a) True topography. Data are assimilated in a swath from approximately $x=0 \mathrm{~km}$ to $x=40 \mathrm{~km}$ in the channel; every other data site is shown. Also, for reference, every other grid cell is shown near open boundary. (b) Estimated topography; $H_{0}=8 \mathrm{~m}$ was the initial guess in this experiment. (c) True topography, as in (a), enlarged to show just the swath where data were assimilated. (d) Estimated topography in the data swath.

guess $H_{0}$ and its nominal or assumed covariance $C^{H}$. We next look more systematically at these two factors.

\section{Specification of priors $H_{0}$ and $C^{H}$}

The first-guess bottom depth $H_{0}$ and its assumed spatial covariance $C^{H}$ are key determinants of the topography estimated from the surface currents. Poor choices of $H_{0}$ can initiate the algorithm in a local minimum of $\mathcal{J}$, making it either difficult or impossible for the algorithm to find an acceptable bottom topography. The spatial structure and smoothness of $C^{H}$ is crucial to regularizing the topographic estimates; without it, nonlinearity causes increasingly smaller scales to arise in the Picard iterates, which cause computational instability. In all cases we wish to avoid generating spurious spatial structure in $H_{\text {est }}$.

The topographic problem is inherently underdetermined, since one generally has thousands of gridded depth values to adjust in order to match a few hundred observations of surface current. It is the choices of $H_{0}$ and $C^{H}$ that remove the indeterminacy. The estimated bottom depth is biased toward $H_{0}$, which would be the expected value of the unknown depth in formal statistical estimation, and the spatial structure of deviations from $H_{0}$ are determined by
$C^{H}$ through Eq. (28). Because there is only one realization of the true bottom depth, the assumptions of statistical estimation cannot be justified, and it is best to regard $H_{0}$ and $C^{H}$ as parameters for regularizing the inversion, removing the indeterminacy in $H$ and biasing its spatial structure according to assumed values of $H_{0}$ and $C^{H}$.

\section{a. First-guess topography $H_{0}$}

In the absence of in situ bathymetry, we have considered two approaches to specifying $H_{0}$. The first approach utilizes morphologic and morphodynamic scaling relationships determined in estuarine and riverine settings. For example, $h(y) \propto y^{-0.8}$, is the relationship between depth $h(y)$ and upstream position $y$ in tidal estuaries (Prandle 2004), and other empirical relationships have been observed in prismatic tidal rivers, alluvial funnel-shaped estuaries, and meandering river systems (e.g., Prandle et al. 2006; Savenije 2005; deSwart and Zimmerman 2009; Frascati and Lanzoni 2009). Note that these scalings are expressed as dimensionally inhomogeneous power laws, but practical application requires that specific, dimensionally consistent formulas be derived for the different morphologic types. Initial efforts in the Haverstraw Bay inversions, discussed below, did utilize a scaling 
relationship expressing a cross-sectional area as a power of distance upstream from the Manhattan Battery, tuned to the known large-scale bathymetry of the Hudson River. Attempts to apply this formalism to other domains demonstrated that it was not generalizable, being overtuned to the Hudson River case. Hence, because of the subjective interpretations of morphologic type, unknown dimensional prefactors, and anthropic influences on morphology (dredging), we do not currently utilize scaling laws to determine $H_{0}$.

A second approach has been found to be more generally applicable. It consists of using the measured currents and a highly reduced dynamics to make a first guess of the bottom topography. The basic approach, formally valid for steady, low Froude number barotropic flow, assumes a balance between the surface pressure gradient and the bottom stress, for example, in the along-channel direction

$$
g D \eta_{y}=-C_{d}\left(u^{2}+v^{2}\right)^{1 / 2} v
$$

Assuming $\eta \ll H$, one may replace $D$ with $H$ and estimate the bottom depth as

$$
H=\frac{C_{d}}{g\left|\eta_{y}\right|}\left(u^{2}+v^{2}\right)^{1 / 2}|v|
$$

In practice, only crude estimates for the pressure gradient are available, so we instead regard it as constant and set the spatial structure of $H$ proportional to $|\mathbf{u}| \max$ $(|u|,|v|)$, where the constant of proportionality is determined by the prescribed maximum depth. In detail, the definition of $H_{0}$ is

$$
\begin{aligned}
\phi(x, y)= & H_{\max } \sqrt{u(x, y)^{2}+v(x, y)^{2}} \\
& \times \max \left[\frac{|u(x, y)|}{u_{\text {max }}^{2}}, \frac{|v(x, y)|}{v_{\max }^{2}}\right] \\
H_{0}(x, y)= & \max \left[\phi(x, y), H_{\min }\right],
\end{aligned}
$$

where spatial dependence on $(x, y)$ has been written explicitly. The function $\max (a, b)$ selects the maximum value of $a$ or $b, H_{\max }$ is the maximum depth (typically obtained at an open-ocean boundary, where $H$ is known), $H_{\min }$ is the minimum depth, and $u_{\max }^{2}$ is given by

$$
u_{\max }^{2}=\sup _{(x, y) \in \mathcal{D}}\left(u^{2}+v^{2}\right)^{1 / 2}|u|
$$

with $v_{\max }^{2}$ defined analogously.

In the applications discussed below, velocity measurements are distributed irregularly in space throughout one or more tidal cycles, so the above method is incomplete. To apply (32) one must obtain $(u, v)$ fields throughout the domain, which is done here by smoothly interpolating the data with (approximately) radial basis functions in space and harmonic functions in time. For the $u$ component of velocity the expansion is

$$
u(x, y, t)=\sum_{k=1}^{M} \sum_{l=1}^{L} \alpha_{k l}^{u} R_{k}(x, y) \exp \left(-i \omega_{l} t\right),
$$

where $k \in\{1, \ldots, M\}$ indexes the $u$ measurement sites, $l \in\{1, \ldots, L\}$ indexes the harmonic basis functions, $\omega_{l}$ is $l$ th frequency (mean and semidiurnal are used), $R_{k}(x, y)=$ $R\left(x-x_{k}, y-y_{k}\right)$ is the basis function associated with measurement site $\left(x_{k}, y_{k}\right)$, and $R(x, y)$ is a bell-shaped Gaussian with correlation scales $\ell_{x}$ and $\ell_{y}$ in the nominal across- and along-channel directions (aligned with the curvilinear coordinates). An analogous expansion is used for $v(x, y)$. The coefficients $\alpha_{\mathrm{kl}}^{u}$ are determined by minimizing the objective function

$$
\mathcal{U}\left(\alpha^{u} ; \gamma\right)=\sum_{k=1}^{M}\left[u\left(x_{k}, y_{k}, t_{k}\right)-u_{k}\right]^{2}+\gamma^{2} \sum_{k=1}^{M} \sum_{l=1}^{L} \frac{\left(\alpha_{\mathrm{kl}}^{u}\right)^{2}}{\beta_{l}^{2}\left(s^{u}\right)^{2}}
$$

which is a function of coefficients $\alpha^{u}=\left\{\alpha_{\mathrm{kl}}^{u}\right\}_{k=1, l=1}^{M, L}$ as well as a regularization parameter $\gamma$, which controls the smoothness of the fit to the measurements. Coefficients $\beta_{l}$ are chosen to partition the variance among the mean and semidiurnal frequencies in the ratio 0.05: 0.8 , and $\left(s^{u}\right)^{2}$ is the mean square of the measurements $\sum\left(u_{k}\right)^{2} / M$. The regularization parameter $\gamma$, which has units of velocity, is selected by minimizing the generalized cross validation estimate of the prediction error (Craven and Wahba 1979; Wahba 1990)

$$
V_{\mathrm{GCV}}^{2}=M^{-1} \sum_{k=1}^{M}\left[u^{(k)}\left(x_{k}, y_{k}, t_{k}\right)-u_{k}\right]^{2},
$$

where $u^{(k)}(x, y)$ is the interpolant (34) computed by minimizing $\mathcal{U}$ excluding the datum at site $k$. In cases with $M>1500$ data sites, the above-described approach is modified to use a reduced basis (Parker 1994) in (34), with all $M$ sites still used in the first term of (35).

To summarize, the first-guess bottom topography is obtained from the measured currents in a two-step process. In the first step, the measurements are mapped onto the model grid by forming a regularized interpolant (or smoother) for the data. The degree of smoothness is controlled by the choice of spatial basis functions and harmonic (constant plus sinusoidal) temporal basis functions, with the fidelity to the data determined by a crossvalidation procedure. In the second step, an assumed 
TABLE 2. First-guess topography parameters.

\begin{tabular}{lll}
\hline \multicolumn{1}{c}{ Parameter } & Symbol & \multicolumn{1}{c}{ Value (range) } \\
\hline Cross-channel correlation scale & $l_{x}$ & $1 \mathrm{~km}(2-4 \Delta x)$ \\
Along-channel correlation scale & $l_{y}$ & $1.75 \mathrm{~km}(2-5 \Delta y)$ \\
Temporal basis functions & $L$ & $3(2-3)$ \\
Temporal priors & $\beta_{l}$ & $0.05,0.8,0.15$ \\
Maximum depth & $H_{\max }$ & $20 \mathrm{~m}(10-25 \mathrm{~m})$ \\
Minimum depth & $H_{\min }$ & $2 \mathrm{~m}(3-10 \mathrm{~m})$ \\
Minimum topographic error & $\Delta_{00}$ & $0 \mathrm{~m}(1-2 \mathrm{~m})$ \\
Fractional topographic error & $\gamma$ & $0.1(0.1-0.5)$ \\
\hline
\end{tabular}

balance between bottom drag and pressure gradient at the time of maximum current is used to transform the gridded currents into bottom depth estimates. Finally, this gridded field is taken as $H_{0}$.

The first-guess topography depends on a number of parameters, in addition to the data values $u_{k}$, which are listed in Table 2. Influential parameters are the spatial scales of the radial basis functions $l_{x}$ and $l_{y}$, which set how the interpolant decays away from data sites. We have had good success with $\left(l_{x}, l_{y}\right) \approx 2(\Delta x, \Delta y)$ in situations with several snapshots of densely spaced measurements. More precise values can be justified by optimizing $V_{\mathrm{GCV}}$ as a function of $\left(l_{x}, l_{y}\right)$, which has also been done and leads to similar values.
Additionally, currents in the Hudson River are dominated by semidiurnal tidal currents, and the partitioning of the variance according to 0.05:0.8 for mean:semidiurnal was guided by an inspection of the currents at several well-sampled sites.

Figure 3 shows an example of first-guess bottom topography computed from AROSS observations at Haverstraw Bay in the Hudson River, bordering New York and New Jersey. Measured surface currents were mapped onto model grid points as shown in Fig. 3a, and the above algorithm was applied using parameter values reported in Table 2 to $H_{0}$ in Fig. 3 b. The good pattern correlation between the true topography $H_{\text {true }}$ and $H_{0}$ is apparent. A quantitative comparison of the two fields, restricted to the region of $(u, v)$ measurements, indicates a root-meansquare (rms) error of $3.3 \mathrm{~m}$ and a correlation coefficient of 0.6 . Figure $3 \mathrm{c}$ shows the generalized cross-validation parameter $V_{\mathrm{GCV}}$ and the rms residual as a function of $\lambda$. The $V_{\mathrm{GCV}}$ statistic selects $\lambda \approx 0.1 \mathrm{~m} \mathrm{~s}^{-1}$ as the optimal value.

Figure 4a shows how the accuracy of $H_{0}$ depends on the quantity and timing of the measured currents with data from the same series of AROSS collections obtained in Haverstraw Bay. The dataset, consisting of 12 separate flyovers, has been subsampled in two ways to demonstrate the dependence on the timing and quantity of data
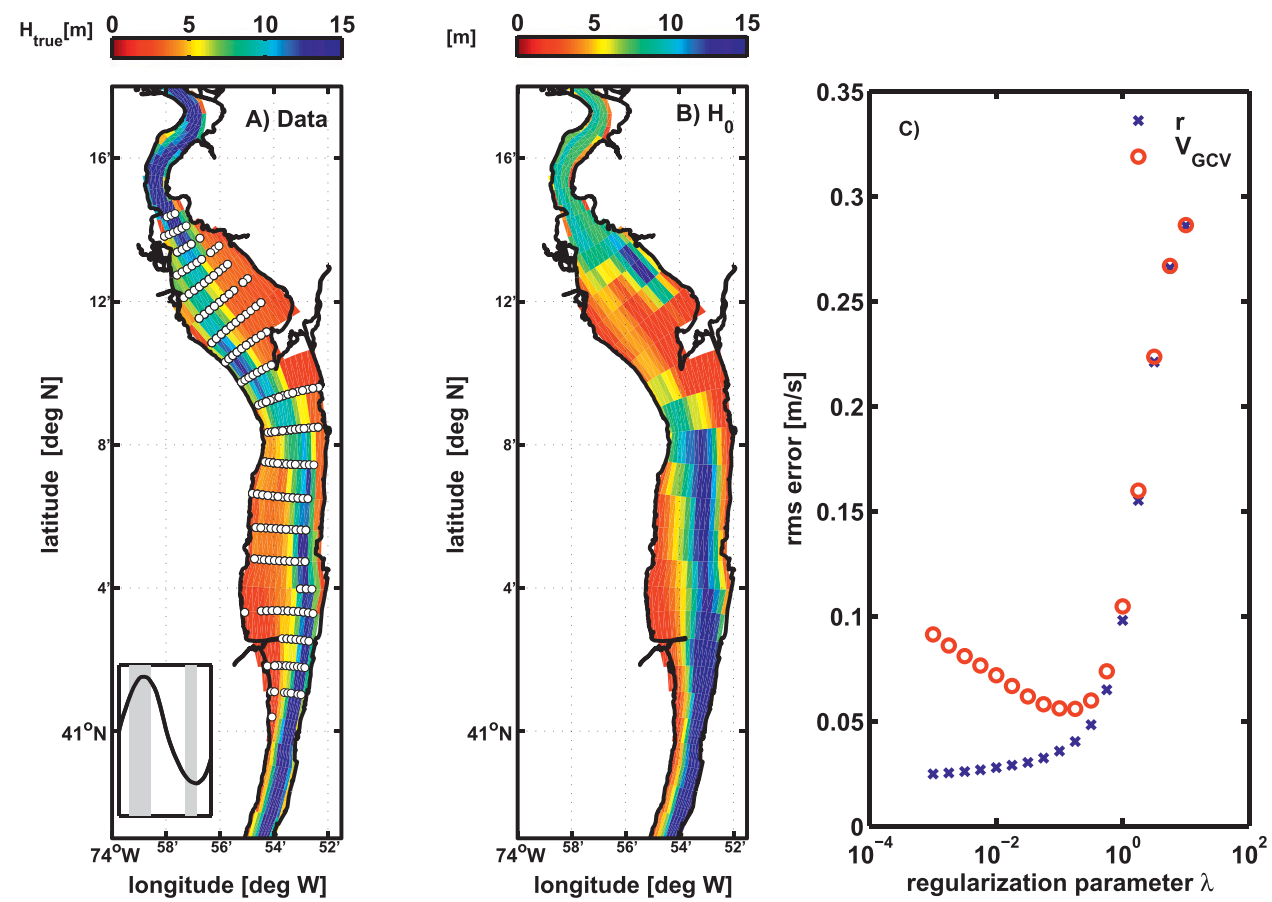

FIG. 3. First-guess topography $H_{0}$ in Haverstraw Bay. (a) Sites of $(u, v)$ data used to compute $H_{0}$; inset shows distribution of data over a tidal cycle. Color scale shows depth of the true topography, which is known for this site in the Hudson River. (b) $H_{0}$ computed via the method described in section 3a. (c) The $r^{2}$ statistic, the first term on the right-hand-side of (35), and the generalized cross-validation statistic $V_{\mathrm{GCV}}$ vary with $\gamma$, the regularization parameter. 

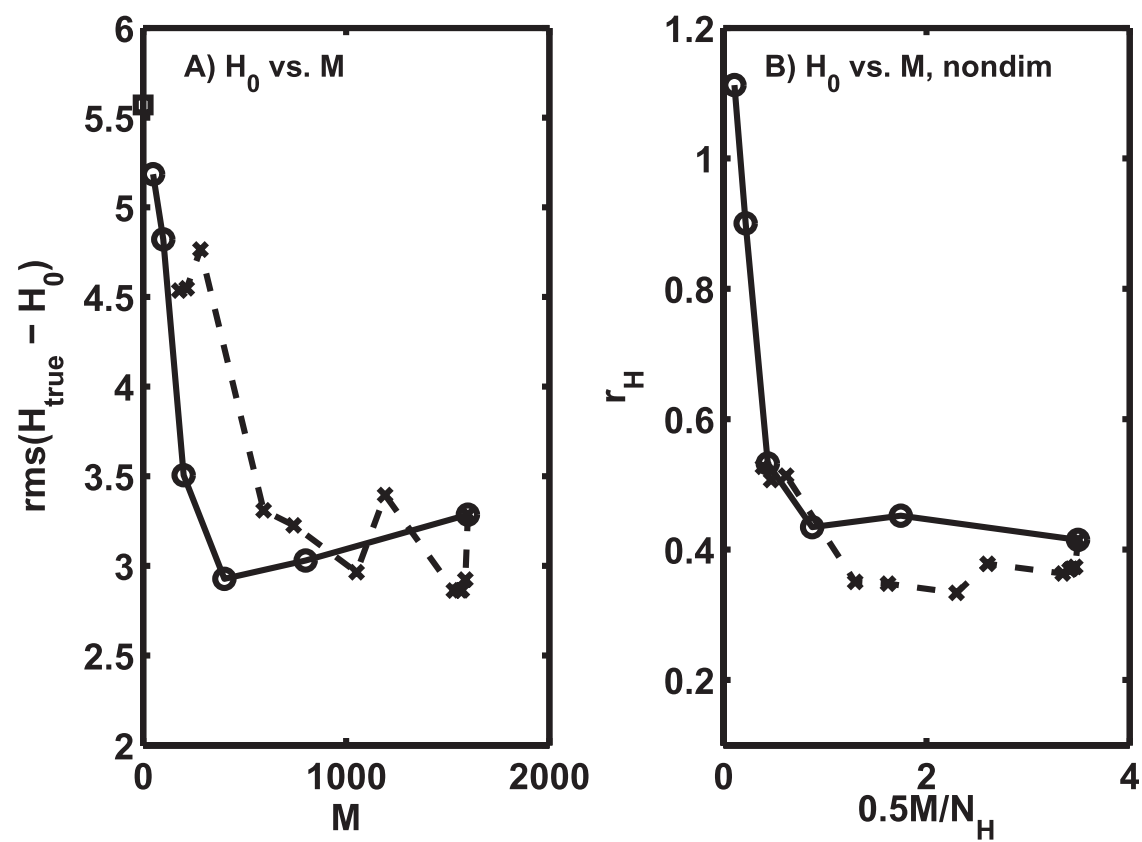

FIG. 4. Dependence of $H_{0}$ on data quantity. The rms error of $H_{0}$ as the number of $(u, v)$ measurements is varied in (a) dimensional and (b) nondimensional form. The rms error when the measurements are uniformly subsampled in space (solid line) and time (circles) are shown, as is the error when the measurements are subsampled in time, where each $\mathrm{x}$ indicates the data accumulated over 15-min intervals (dashed line). (b) The curves in (a) collapse when the rms error is nondimensionalized using the kinetic energy of the measured currents, and the first guess is not substantially improved by taking more than a single snapshot of the domain, $0.5 M / N_{H}>1$.

as measured by the rms error in topography $\left\langle\left(H_{\text {true }}-\right.\right.$ $\left.\left.H_{0}\right)^{2}\right\rangle^{1 / 2}$, where brackets indicate spatial average over Haverstaw Bay $\left(41^{\circ} 1^{\prime}-41^{\circ} 14^{\prime}\right)$. In the first case (dashed line) the data are accumulated from 15-min windows. One can see that the error in $H_{0}$ with a single window of data is about $4.6 \mathrm{~m} \mathrm{rms}$. As subsequent data windows are included, the error decreases to between 3 and $3.5 \mathrm{~m} \mathrm{rms}$. The second way of subsampling is shown by the solid line, in which the entire dataset has been thinned uniformly in space and time. A rapid decrease in error occurs with increasing data. In the absence of any data, $M=0$, we have taken $H_{0}=H_{\min }$, with an rms error of $5.6 \mathrm{~m}$.

Figure $4 \mathrm{~b}$ shows the same data as those in Fig. 4a, except that the values have been nondimensionalized. The rms error in $H_{0}$ is scaled by a term proportional to the average kinetic energy of the measured currents

$$
r_{H}=\frac{g H_{\min }}{q} \frac{F r_{0}^{2}}{C_{d}} \frac{\left\langle\left(H_{\text {true }}-H_{0}\right)^{2}\right\rangle^{1 / 2}}{\left\langle\left(H_{\text {true }}-H_{\text {min }}\right)^{2}\right\rangle^{1 / 2}}
$$

where $\mathrm{Fr}_{0}=10^{-2}$ is a nominal Froude number and $q=M^{-1} \sum u_{k}^{2}$ is twice the average kinetic energy of the measurements. Also, the number of measurements $M$ is nondimensionalized by $N_{H}=230$, the number of $H$ grid cells in Haverstraw Bay. For small $M$ one can see that the difference between the two subsetting methods collapses, which is consistent with the scaling $H_{0} \propto q$ suggested by (31). Measurements during times of peak currents have more favorable signal-to-noise ratios than measurements at other times.

Figure 4 also shows that the accuracy of $H_{0}$ is little improved by making more than one spatial snapshot. For $M>2 N_{H}$ there is no systematic reduction in the rms error of $H_{0}$. A similar conclusion could be obtained from plots of the $\left(H_{\text {true }}, H_{0}\right)$ correlation coefficient, which attains a maximum value of about 0.7 when $M=2 N_{H}$. For $M<2 N_{H}$, there is a suggestion of $M^{-1 / 2}$ scaling for the rms error, but for $M>2 N_{H}$ systematic error dominates. Presumably the systematic errors are due to incorrect values of $H_{\max }$ and $H_{\min }$ in (32), finite Froude number or nonuniform pressure gradient effects in (31), and mapping error resulting from the assumed parameterization (34).

\section{b. Spatial covariance $C^{H}$}

The spatial autocovariance of the bottom topography is modeled as 


$$
\begin{aligned}
c^{H}\left(x_{1}, y_{1}, x_{2}, y_{2}\right)= & \Delta H\left(x_{1}, y_{1}\right) \Delta H\left(x_{2}, y_{2}\right) \\
& \times c^{H}\left(x_{1}, y_{1}, x_{2}, y_{2}\right),
\end{aligned}
$$

where $c^{H}\left(x_{1}, y_{1}, x_{2}, y_{2}\right)$ is a positive, definite correlation function, and $\Delta H$ is a spatially dependent function, nominally, the standard error of $H_{0}$. The spatial correlation function has been chosen with a bias toward smoothness,

$$
c^{H}\left(x_{1}, y_{1}, x_{2}, y_{2}\right) \approx \exp \left[-\frac{\left(x_{1}-x_{2}\right)^{2}}{2 L_{x}^{2}}-\frac{\left(y_{1}-y_{2}\right)^{2}}{2 L_{y}^{2}}\right],
$$

where $L_{x}$ and $L_{y}$ are the across- and along-channel correlation scales. The above expression is approximate because it does not satisfy boundary conditions. In practice, $c^{H} \circ \lambda^{H}$ is computed by time stepping a diffusion equation (Derber and Rosati 1989; Weaver and Courtier 2001) with boundary conditions that are appropriate to maintain positive definiteness on bounded irregular domains (either Neumann or Robin boundary conditions may be selected).

More complex models of the spatial correlation have been contemplated, but in the absence of compelling arguments we use (38). In the case of Haverstraw Bay, the spatial cross correlation of $H_{0}$ and $H_{\text {true }}$ can be computed, but the results are noisy and suggest $L_{x} \approx 1 \mathrm{~km}$ and $L_{y} \approx$ $10 \mathrm{~km}$ from the zero crossings of the sample correlation. In an effort to develop a more refined state-dependent model, the method of Riishojgaard (1998) was implemented in which the minor axis of correlation is aligned with $\nabla H_{0}$. This approach magnified spurious features in the first guess, and it has not been pursued.

It has been found useful to treat $\Delta H$ as a statedependent function, in the sense that it depends on $\bar{H}^{n}$ at each Picard iteration. We choose a linear function,

$$
\Delta H=\Delta_{00}+\gamma \bar{H}^{n},
$$

for constants $\Delta_{00}$ and $\gamma$, which permits larger topographic correction in deep water. Once again, there are insufficient data to make a strong argument for this form, but it is not contradicted by the empirical spread of $H_{0}$ versus $H_{\text {true }}$ values, which show errors increasing with depth.

\section{Proof-of-concept demonstration: Haverstraw Bay, Hudson River, New York}

To provide a proof of concept, the preceding formulation has been applied to a river estuary system for which the bottom topography is well known from in situ observations.
On 15 October 2008, from 1300 to 2100 UTC, surface current data were collected by the AROSS remote sensing system described in Dugan et al. (2001b). The raw observations consisted of vector currents on a grid of approximately $128 \mathrm{~m} \times 128 \mathrm{~m}$ horizontal resolution, with gaps caused by boat wakes and other image anomalies. A series of airborne collections were obtained, which may be regarded as 12 synoptic snapshots of surface currents. Preprocessing data for assimilation consisted of applying an empirically derived scaling to convert the surface current into an equivalent depth average current, with the coefficient being obtained from a linear regression of surface and depth average currents simulated by the New York Harbor Observation and Prediction System (NYHOPS; Bruno et al. 2006). The observed currents were mapped onto the hydrodynamic model grid, rotating the vectors from geographic coordinates into the curvilinear coordinate system of the model, and the observation kernels $\left(\mu_{i}, \nu_{i}\right)$ were defined as spatial averages over grid cells at each observation time. Figure 3 a shows the locations of all of the observations, and it can be seen that they cover a swath approximately $6 \mathrm{~km}$ wide. Figure 5 shows a representative sample of individual snapshots, which illustrates the nonuniform temporal sampling. Each dot corresponds to up to four individual measurements that have been averaged onto the computational grid.

Measurement error consists of instrumentation error in the raw currents, processing error in the transformation of the surface to the depth average current, remapping error from mapping and averaging observations onto model grid nodes, and errors of representation. The instrumentation error is approximately $0.1 \mathrm{~m} \mathrm{~s}^{-1}$ and $5^{\circ}$ for current speed and direction, respectively, which are values determined by independent calibration of the AROSSmeasured surface currents (J. Dugan 2010, personal communication; Piotrowski and Dugan 2002). The processing error is between 0.1 and $0.15 \mathrm{~m} \mathrm{~s}^{-1}$, as determined by the residual of the surface to the depth average current regression. Remapping errors are believed to be less than $0.01 \mathrm{~m} \mathrm{~s}^{-1}$ because of the similarity of the observational and numerical model grids. Representation error is caused by signals in the observations resulting from boundary currents or transient eddies that are not resolved in the numerical model; intercomparison of the observed currents with NYHOPS model simulations (which utilizes the known bathymetry of the Hudson River) suggests that the representation error is approximately $0.1 \mathrm{~m} \mathrm{~s}^{-1}$. It is difficult to make any of these error estimates more precise, and, in fact, the $V_{\mathrm{GCV}}$ statistic shown previously suggests that the instrumental error might be smaller, about $6 \mathrm{~cm} \mathrm{~s}^{-1}$ (cf. Fig. 3). In light of these considerations we adopt an error model consisting of $0.1 \mathrm{~m} \mathrm{~s}^{-1}$ for speed 

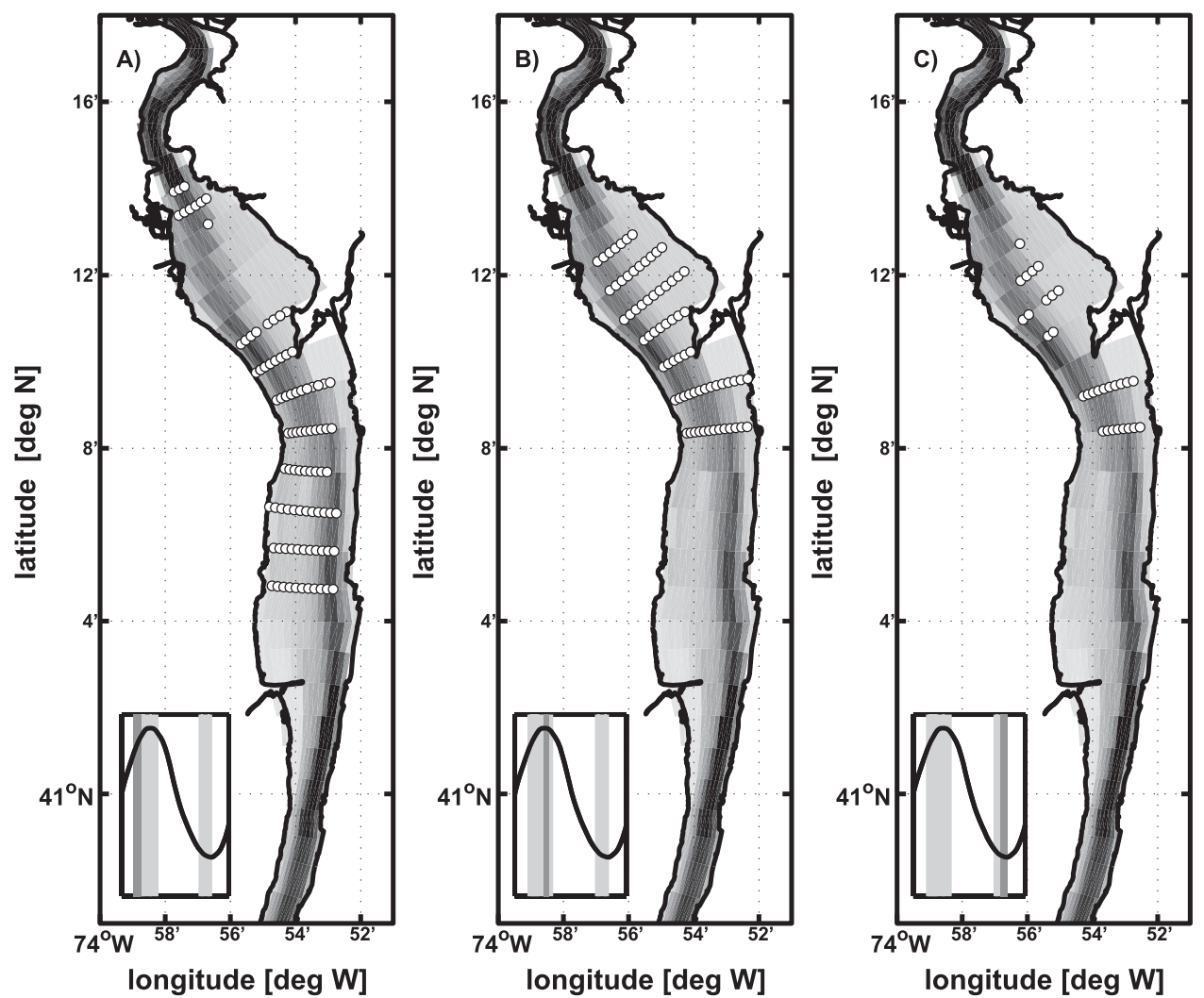

FIG. 5. Haverstraw Bay measurements. Locations of surface current measurements at (left to right) three representative times are shown. AROSS data have been averaged onto model grid cells, and only sites of $v$ component velocity are shown. For reference, the inset shows the water elevation from 1200 to 2400 UTC 15 Oct 2008, with the time of the observations (dark vertical line) and times of other observations (light shading) shown; vertical range is $\pm 0.75 \mathrm{~m}$.

and $5^{\circ}$ for direction. This representation accommodates separate assumed errors for the across- and along-channel components of velocity, which are generally aligned with the model grid.

The first guess for bottom topography $H_{0}$ and its error covariance $C^{H}$ were discussed in section 3 , above. The inversion shown below uses the values $L_{x}=0.5 \mathrm{~km}$ and $L_{y}=3 \mathrm{~km}$ for the $C^{H}$ correlation scales, and $\Delta H$ is set with state-dependent formula [Eq. (39)], taking $\Delta_{00}=$ 0 and $\gamma=0.1$, with the latter being comparable to the fractional error in the velocity measurements.

The results of the inversion are summarized in Fig. 6. Because the corrections to the topography are predominantly local to the observation sites, the quantitative comparisons have been restricted to the grid nodes containing velocity measurements. Likewise, the figures display Haverstraw Bay, although the modeled domain extends $270 \mathrm{~km}$, starting from Manhattan Battery, and ending at Troy, New York.

Figure 6a illustrates the true Haverstraw Bay topography, which contains a single channel that crosses the Bay from east to west, going northward. The depth varies from over $20 \mathrm{~m}$, at the north and south ends of the Bay, to shoals only a few meters deep (truncated at $2 \mathrm{~m}$ in the numerical model). The first-guess topography (Fig. 6b) shows a similar structure, but the depth of the channel is reduced between $41^{\circ} 8^{\prime}$ and $41^{\circ} 13^{\prime}$. Also, $H_{0}$ contains a spurious deep area located east of the true channel at the north end of the Bay. Analysis of the model fields has indicated a phase lag in current between the shoals and deeper areas, and this anomalous pit in the north is due to ill-timed and sparse data over the shoals.

The topography obtained by nonlinear data assimilation is shown in Fig. 6c. It has improved the first-guess significantly in terms of both topographic accuracy, which can be computed since the topography is known here, and in terms of predicted currents. Figure 7 compares the actual and estimated topography at the grid nodes where $(u, v)$ measurements were obtained. The rms error in the first guess is $3.6 \mathrm{~m}$, which is reduced to $2.5 \mathrm{~m}$ in the optimized topography. Residuals in the currents are reduced from rms values of $(9,16)$ to $(7,10) \mathrm{cm} \mathrm{s}^{-1}$, for the $(u, v)$ 

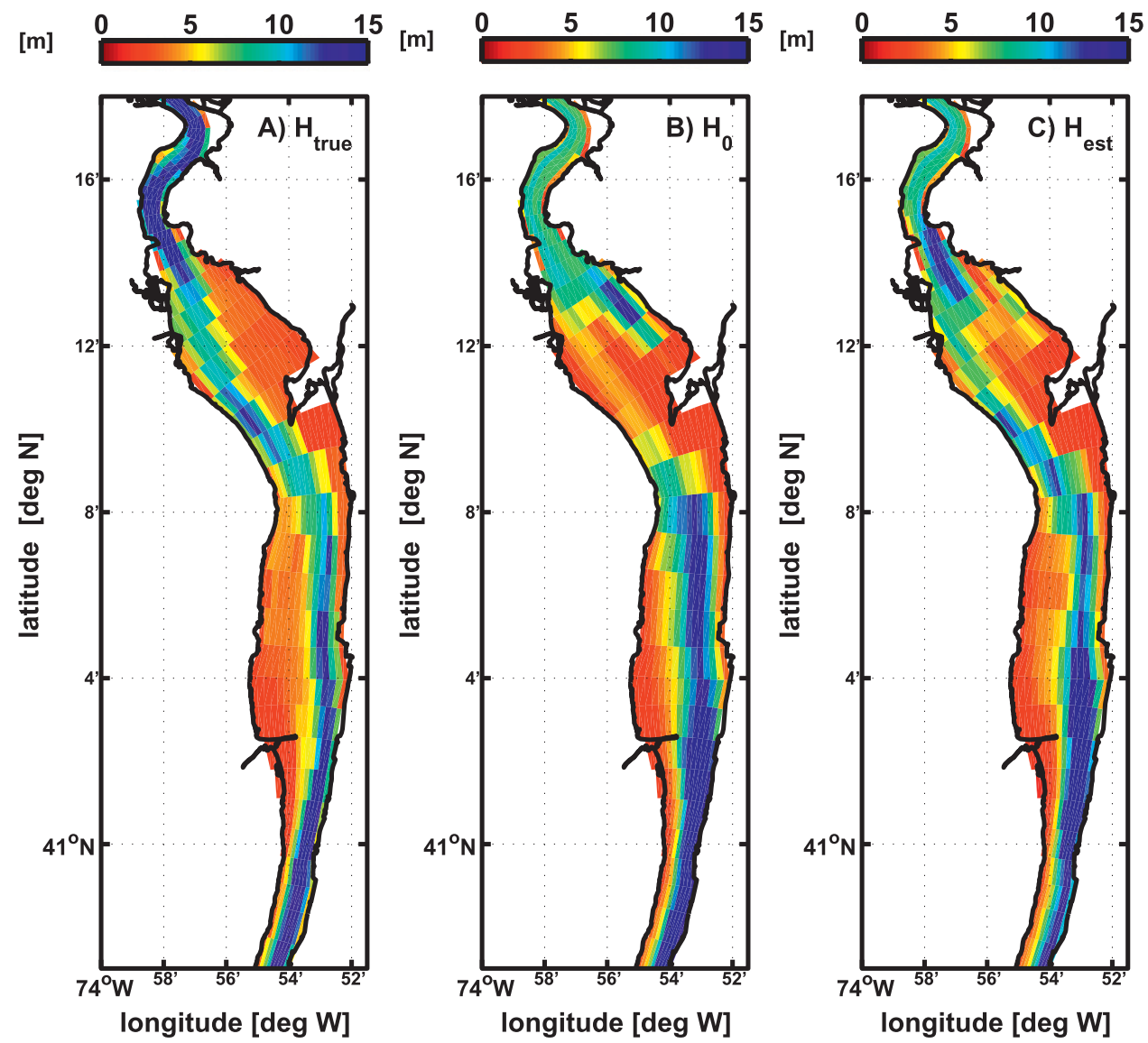

FIG. 6. Haverstraw Bay topography. (a) The known topography $H_{\text {true }}$ obtained from in situ bathymetric surveys. (b) First-guess topography $H_{0}$ computed from the AROSS measurements as described in section 3a. (c) Estimated topography $H_{\text {est }}$ obtained after two Picard iterations.

velocity components, with the latter values being comparable to measurement error.

The value of the optimized objective function provides a basis for assessing the consistency of the hypothesized dynamics and error models. If the hypothesized priors are correct, and the errors are normally distributed, the optimized value of $\mathcal{J}$ is a $\chi^{2}$ variable with $M$ degrees of freedom. Hence, the expected value of $\mathcal{J}$ is $M$, where $M=1314$ in this case. Figure 8 plots the optimized value of $\mathcal{J} / M$ for the 10 Picard iterates computed (solid line, circles). One can see that $\mathcal{J} \approx M$ for the second Picard iteration, which suggests that this solution is consistent with the hypothesized errors, and it contains the smoothest topography necessary to explain the surface current measurements. Figure 8 also shows the error compared to the known topography (dashed line, squares). One sees that the accuracy of the topography changes little between Picard iterates 1-6, which is consistent with the comparison between iterates 1 and 2 shown previously in Fig. 7 . The value of $\mathcal{J} / M$ is decreased in subsequent iterates, but one sees that the rms topography error increases. This figure demonstrates that extending the Picard iteration to further minimize $\mathcal{J}$ is futile, because one would add spurious structure to the topography merely to fit noise in the data.

\section{Discussion}

Whereas previous attempts at topographic estimation by variational data assimilation have either relied on reductions in the degrees of freedom to be estimated (Heemink et al. 2002) or have considered idealized problems where the number of data were equal to the number of depth grid points (Losch and Wunsch 2003), the present approach finds an optimal estimate of bottom depth in computational domains of arbitrary size, using actual data. Of course, the identifiability of the bottom topography conforms to the general principle that the number of degrees of freedom that can be identified are equal to the number of independent data. The estimation procedure is successful because, at each linear iteration step, bottom topography is optimized over just the observable degrees of freedom. 

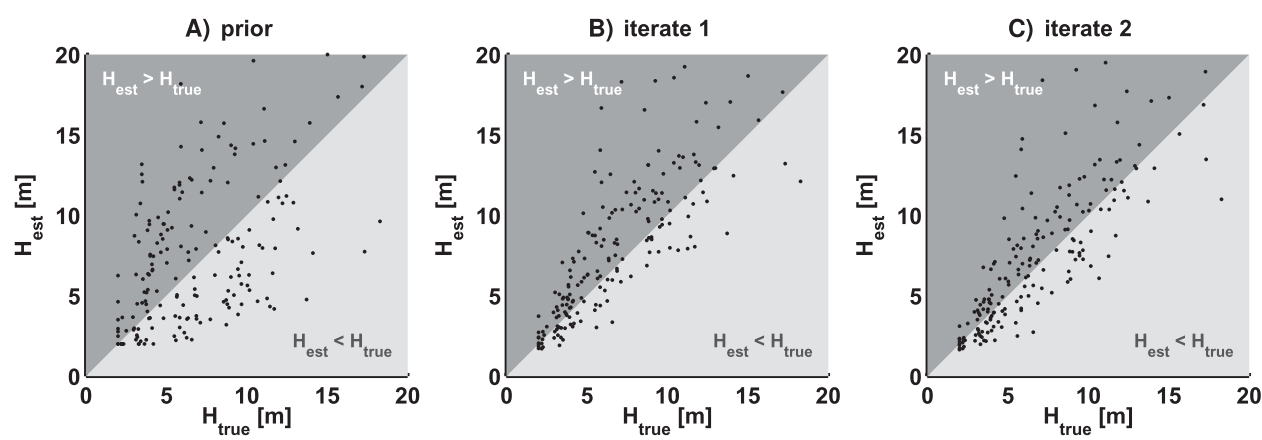

FIG. 7. Topographic error vs depth. Estimated depth is plotted as a function of the true depth at grid nodes within Haverstraw Bay. The shading for $H<H_{\text {true }}$ (light gray) and $H>H_{\text {true }}$ (dark gray) classify the depth errors, according to the hypothetical impact on navigation safety, which strongly favors errors of the form $H<H_{\text {true }}$ (left) Large scatter of the first-guess $H_{0}$ compared to true topography $H_{\text {true }}$; correlation is 0.6. (middle) Scatter is largely reduced after a single Picard iteration and (right;) there is very little change from iteration 1 . Correlation with true topography is 0.8 for iteration 2 .

Nonetheless, the estimated topography depends on a large number of factors including the parameters of the solution algorithm, the covariance $C^{H}$, the geometry of the domain, the distribution of observations in space and time, etc. This section considers several factors that influence the accuracy and utility of the estimated topography.

\section{a. Timing and accuracy of observations}

Because of the presence of background currents $(\bar{u}, \bar{v})$ in the topography updating Eq. (28), the assimilation accuracy is dependent on the timing of the observations relative to the strength of the background currents. In tidally forced estuaries, for example, this suggests that the currents ought to be measured during the times of peak ebb or flood, if possible. This result is foreshadowed by Fig. 4, where it was shown that, for small $M$, the accuracy of the first guess is related to kinetic energy of the observed flow.

Figure 9a illustrates the impact of data timing obtained from identical twin experiments in the idealized channel model (section 2c) using a single snapshot of observations. The observation time is varied in 1-h increments, denoted 1-7 in the figure. Error is plotted as a function of the average kinetic energy to emphasize the timing relative to the tidal cycle. One sees that the relationship between accuracy and timing of observation is not as simple as for $H_{0}$, and 1-h changes in the observation time lead to more than factor of 2 changes in error (e.g., going from times 3 to 4 and from 6 to 5). In fact, the data do collapse to nearly a straight line if the error is plotted as a function of kinetic energy leading the observations by $1 \mathrm{~h}$. As might be anticipated, results depend on the phases of $\nabla \bar{\eta}$ and $(\bar{u}, \bar{v})$ relative to the observations in (28).

Figure $9 \mathrm{~b}$ shows how topography error depends on the measurement error in the same idealized model. The error
$\left(H_{\text {est }}-H_{\text {true }}\right)$ is proportional to $\sigma_{u}$, except for both large and small values. At the extremes of the range of $\sigma_{u}$ the error in the estimated topography is determined by the formally unobservable, or unidentifiable, degrees of freedom. Thus, for small $\sigma_{u}$, the error is determined by the topography away from the observed swath, where terms in (28) are small (where the topography is unobservable by surface currents). For large $\sigma_{u}$, the data have little impact, and the error is determined by the first guess.

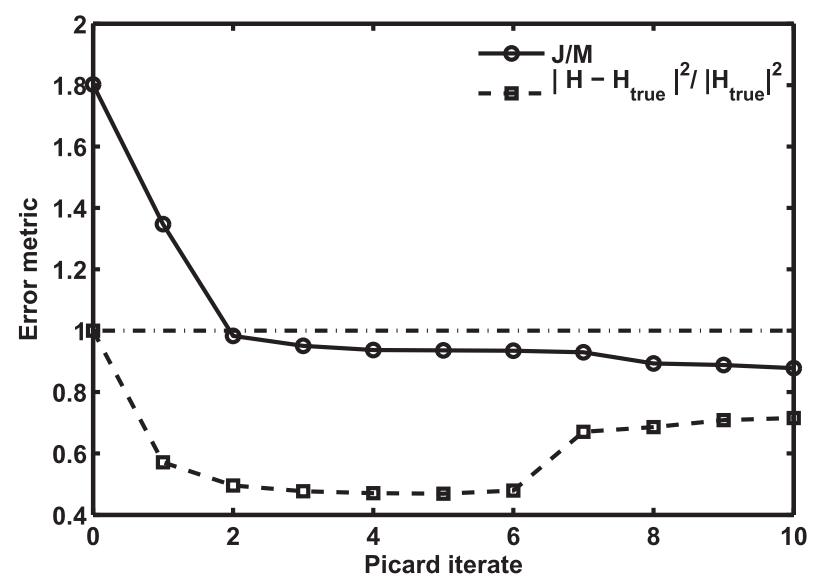

FIG. 8. Errors vs Picard iterate. The value of the optimized objective function scaled by the number of measurements, $\mathcal{J} / M$ is shown with the solid line (circles) as a function of Picard iteration. Iteration 0 corresponds to no assimilation. The expected value of $\mathcal{J} / M$ is 1 (dash-dot line), which is achieved for the second Picard iteration, and indicates that the estimated topography and hydrodynamic model explain all the nonnoise variance in the measurements. The rms residual between the estimated (dashed line) and true topography (squares) is shown. One sees that the rms error does decrease very slightly from Picard iteration 2 to 4, but it increases after that (indicating overfitting of velocity data for these and subsequent Picard iterations). 

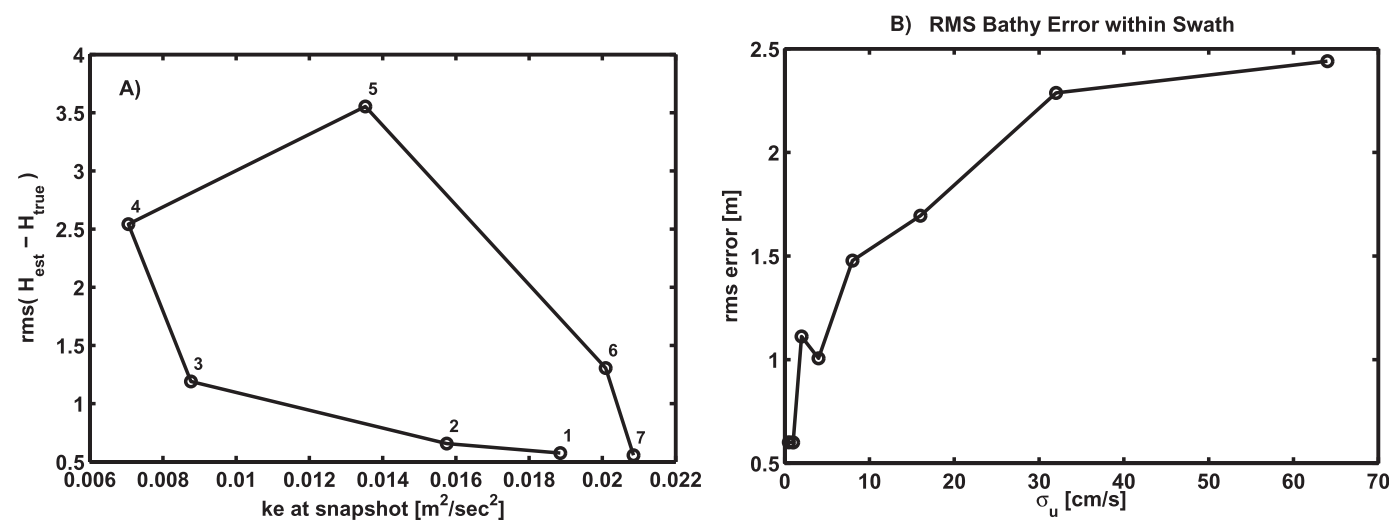

FIG. 9. Sensitivity to measurement time and accuracy. Panels indicate how the rms error of the estimated topography, $\left\langle\left(H_{\text {est }}-H_{\text {true }}\right)^{2}\right\rangle^{1 / 2}$, depends on measurement (a) timing and (b) error in the idealized channel model (see section 2c). (a) Error at seven different measurement times separated by $1 \mathrm{~h}$ during half a tidal cycle (dots, 1-7). Kinetic energy is shown on the $x$ axis to emphasize timing relative to maximum current. (b) Error in the estimated topography as a function of measurement error $\sigma_{i}=\sigma_{u}$.

\section{b. Parameter dependence}

The estimated topography depends on $L_{x}, L_{y}, l_{x}$, and $l_{y}$, which are unknown parameters that control the spatial correlation of the topography. Although experience is limited, we have found that even extreme misspecifications of these parameters lead to, at most, factor of 2 degradations compared to optimal values. Respectively, $L_{x}$ and $L_{y}$ are the across- and along-channel correlation scales of the topographic corrections, and it is essential that these be greater than grid spacing in respective directions; $l_{x}$ and $l_{y}$ play a similar role in determining the first guess $H_{0}$, and they should be at least as large as the average spacing between the data in the respective directions.

Another level of parametric dependence to consider is the form of the first-guess topography (32). It may be possible to obtain an improved first guess using other techniques to smoothly grid the measured currents. For example, Vennell and Beatson (2006) formulate the gridding of sparse vector currents as a streamfunction estimation problem. While their approach discards information in the divergent part of the horizontal velocity, it may provide a more stable and less parameterized alternative to the procedure described in section $3 a$, possibly interpolating better through data voids.

The estimated topography also depends on the values of $H_{\min }$ and $H_{\max }$ that are used in the first-guess parameterization, which are set equal to the minimum and maximum depth, respectively, on the open boundary. Experiments have been conducted using the idealized channel model of section $2 \mathrm{c}$ by simply taking $H_{0}=H_{00}$, a constant value. In these experiments it was found that varying $H_{00}$ between $H_{\min }$ and $H_{\max }$ led to factor of 3 changes in the rms error in $H_{\text {est }}$, and it is believed that the values of $H_{\min }$ and $H_{\max }$ represent the largest source of uncertainty in the first guess in realistic domains.

\section{c. Nonlocal effects}

Equations (23)-(27) indicate that information is carried through the domain by gravity wave dynamics subject to bottom friction, but frictional damping is not small, as evidenced by the success of the reduced dynamics in section 3a. Bottom friction makes the assimilation problem largely local, because information cannot propagate far from measurement sites before being damped and lost from the system. Away from the measurements the corrections to the first-guess topography are generally small, exceptions occurring, for example, at narrows or flow constrictions where changes in depth strongly influence tidal transport and currents throughout the river.

A canonical decomposition (McIntosh 1987; Bennett 1992) of the data assimilation system has been used to quantify the stability of the inverse and analyze the topographic degrees of freedom constrained by the current measurements. Because the inversion problem is nonlinear, the canonical decomposition is applied to the linear system defined by the final Picard iteration. The results of this analysis when applied to Haverstraw Bay agree with the qualitative comments above. The depth estimates depend most strongly on nearby data, with the domain of influence determined by $L_{x}$ and $L_{y}$. The inversion is stable, with $N_{s} \approx 40$ significant degrees of freedom, where $N_{s}$ is approximately equal to the area of Haverstraw Bay divided by the area of influence, $L_{x} \times$ $L_{y}$. There is substantial redundancy in the measurement array, which contributes to good stability of the identified topography. 


\section{Summary}

Accurate hydrodynamic modeling of rivers, bays, estuaries, etc., requires that bottom topography be known. In many places it is not practical to obtain in situ bathymetry on a regular basis sufficient to resolve time-varying bed forms or channel in fill; also, natural and anthropic hazards may make it too dangerous or expensive to conduct in situ surveys at the desired spatial resolution. In these situations is it desirable to estimate bottom topography from remotely sensed information.

The results of this paper demonstrate that bottom topography may be estimated using methods of variational data assimilation. Remotely sensed surface currents, obtained from an analysis of georeferenced airborne imagery (Dugan et al. 2001b), have been assimilated into a barotropic shallow-water model. A solution technique has been developed using extensions of linear variational data assimilation methods. The practicability of the method depends on the geometry of the domain and dynamical balances involved, but we find that the equivalent of 40-120 model integrations is sufficient to stably estimate the topography near the site of the remotely sensed observations. Depending on the application, fewer (e.g., 20) model integrations may be necessary if a less precise estimate is required.

It is assumed that the bottom topography and currents are coupled by barotropic shallow-water dynamics. The measured surface currents are transformed into equivalent vertical average currents using an empirical scaling, close to what is obtained using unstratified law-ofthe-law-type dynamics. In principle, stratified threedimensional dynamics could be used directly as the basis for the estimation of bottom topography, but this would require enough data to well constrain the baroclinic processes. Without in situ data to assist in estimating the baroclinic pressure gradient, attempts at using threedimensional assimilation seem premature, and our experience indicates that barotropic dynamics are adequate for applications to tidally forced rivers and estuaries. Hence, the three-dimensional coupling is left as an avenue of future research.

The methodology employed is applicable with other forms of data, for example, water elevation or tide gauge measurements, and with models of larger geographic extent. Application to smaller scales where there is significant wetting and drying, or where advection dominates waves (e.g., high Froude number dynamics), may prove more challenging because these constraints add additional nonlinearity. Wetting and drying algorithms are generally not differentiable (Ji et al. 2001), so the variational methods that are used here may need to be augmented with inequality constraints.
Acknowledgments. The views expressed here are those of the authors and do not reflect the official policy or position of the Department of Defense or the U.S. Government. This project was funded by the U.S. Defense Advanced Research Projects Agency (DARPA)/Naval Research Laboratory Contract N00173-07-C-6001. The NYHOPS system was developed with support from the Office of Naval Research Grant N00014-03-1-0633. Arete Associates provided the surface current observations used in this project, and we gratefully acknowledge their support and participation. This project utilized the Inverse Ocean Model software system, developed with support from the U.S. National Science Foundation, Grant 0121542.

\section{REFERENCES}

Bennett, A. F., 1992: Inverse Methods in Physical Oceanography. 1st ed. Cambridge University Press, 346 pp.

- 2002: Inverse Modeling of the Ocean and Atmosphere. Cambridge University Press, $234 \mathrm{pp}$.

—, B. S. Chua, B. L. Pflaum, M. Erwig, Z. Fu, R. D. Loft, and J. C. Muccino, 2008: The Inverse Ocean Modeling system. Part I: Implementation. J. Atmos. Oceanic Technol., 25, 16081622.

Blumberg, A. F., and H. J. Herring, 1987: Circulation modelling using orthogonal curvilinear coordinates. Three-Dimensional Models of Marine and Estuarine Dynamics, J. C. J. Nihoul and B. M. Jamart, Eds., Elsevier Oceanography Series, Vol. 45, Elsevier, 55-88.

__ , and G. L. Mellor, 1987: A description of a three-dimensional coastal ocean circulation model. Three-Dimensional Coastal Ocean Models, N. S. Heaps, Ed., Amer. Geophys. Union, $1-16$.

—_ and N. Georgas, 2008: Quantifying uncertainty in estuarine and coastal ocean circulation modeling. J. Hydraul. Eng., 134, 403-415.

Brent, R. P., 1973: An algorithm with guaranteed convergence for finding a zero of a function. Algorithms for Minimization without Derivatives, Prentice Hall, 47-60.

Bruno, M. S., A. F. Blumberg, and T. O. Harrington, 2006: The urban ocean observatory-Coastal ocean observations and forecasting in the New York Bight. J. Mar. Sci. Environ., C4, 31-39.

Chua, B., and A. F. Bennett, 2001: An inverse ocean modeling system. Ocean Modell., 3, 137-165.

Courtier, P., 1997: Dual formulation of four-dimensional assimilation. Quart. J. Roy. Meteor. Soc., 123, 2449-2461.

Craven, P., and G. Wahba, 1979: Smoothing noisy data with spline functions: Estimating the correct degree of smoothing by the method of generalized cross-validation. Numer. Math., 31, $377-403$.

Cressman, G., 1959: An operational objective analysis system. Mon. Wea. Rev., 87, 367-374.

Das, S. K., and R. W. Lardner, 1991: On the estimation of parameters of hydraulic models by assimilation of periodic tidal data. J. Geophys. Res., 96, 15 187-15 196.

Derber, J., and A. Rosati, 1989: A global oceanic data assimilation system. J. Phys. Oceanogr., 19, 1333-1347.

de Sturler, E., 1994: Iterative methods on distributed memory computers. Ph.D. thesis, Delft University of Technology, 130 pp. 
deSwart, H. E., and J. T. Zimmerman, 2009: Morphodynamics of tidal inlet systems. Annu. Rev. Fluid Mech., 41, 203-229.

Dugan, J. P., and C. C. Piotrowski, 2003: Surface current measurement using visible image time series. Remote Sens. Environ., 1, 309-319.

— sensing of ocean waves. J. Atmos. Oceanic Technol., 18, 12671276.

_ C. C. Piotrowski, and J. Z. Williams, 2001b: Water depth and surface current retrievals from airborne optical measurements of surface gravity wave dispersion. J. Geophys. Res., 106, 16 903-16 915.

Frascati, A., and S. Lanzoni, 2009: Morphodynamic regime and long-term evolution of meandering rivers. J. Geophys. Res., 114, F02002.

Heemink, A. W., E. E. Mouthaan, M. R. Roest, E. A. Vollebregt, K. B. Robaczewska, and M. Verlaan, 2002: Inverse 3D shallow water flow modeling of the continental shelf. Cont. Shelf Res., 22, 465-484.

Hirose, N., 2005: Least-squares estimation of bottom topography using horizontal velocity measurements in the Tsushima/Korea Straits. J. Oceanogr., 61, 789-794.

Ji, Z., M. R. Morton, and J. M. Hamrick, 2001: Wetting and drying simulation of estuarine processes. Estuarine Coastal Shelf Sci., 53, 683-700.

Lardner, R. W., A. H. Al-Rabeh, and N. Gunay, 1993: Optimal estimation of parameters for a two-dimensional hydrodynamical model of the Arabian Gulf. J. Geophys. Res., 98, 18 229-18 242.

Lilly, D. K., 1967: The representation of small-scale turbulence in numerical simulation experiments. Proc. IBM Scientific Computing Symp. Environmental Science, IBM, 195.

Losch, M., and C. Wunsch, 2003: Bottom topography as a control variable in an ocean model. J. Atmos. Oceanic Technol., 20, 1685-1696.

McIntosh, P., 1987: Systematic design of observational arrays. J. Phys. Oceanogr., 17, 885-902.

Mourre, B., P. De Mey, F. Lyard, and C. Le Provost, 2004: Assimilation of sea level data over continental shelves: An ensemble method for the exploration of model errors due to uncertainties in bathymetry. Dyn. Atmos. Oceans, 38, 93-121.

Muccino, J. C., and Coauthors, 2008: The inverse ocean modeling system. Part II: Applications. J. Atmos. Oceanic Technol., 25, 1623-1637.

Parker, R. L., 1994: Geophysical Inverse Theory. Princeton University Press, $400 \mathrm{pp}$.

Piotrowski, C. C., and J. P. Dugan, 2002: Accuracy of bathymetry and current retrievals from airborne optical time-series imaging of shoaling waves. IEEE Trans. Geosci. Remote Sens., 40, 2606-2618.

Prandle, D., 2004: How tides and river flows determine estuarine bathymetries. Prog. Oceanogr., 61, 1-26.

—, A. Lane, and A. J. Manning, 2006: New typologies for estuarine morphology. Geomorphology, 81, 309-315.

Riishojgaard, L. P., 1998: A direct way of specifying flow-dependent background error correlations for meorological analysis systems. Tellus, 50A, 42-57.

Sarkisyan, A. S., and V. F. Ivanov, 1971: Joint effect of baroclinicity and bottom relief as an important factor in the dynamics of sea currents. Bull. Acad. Sci. USSR Atmos. Oceanic Phys., 7, 173-188.

Savenije, H. H., 2005: Salinity and Tides in Alluvial Estuaries. Elsevier, 194 pp.

Smagorinsky, J., 1963: General circulation experiments with the primitive equations I. The basic experiment. Mon. Wea. Rev., 91, 99-164.

Ten-Brummelhuis, P. G., A. W. Heemink, and H. van den Boogard, 1993: Identification of shallow sea models. Int. J. Numer. Methods Fluids, 17, 637-665.

Vennell, R., and R. Beatson, 2006: Moving vessel acoustic Doppler current profiler measurements of tidal stream function using radial basis functions. J. Geophys. Res., 111, C09002, doi:10.1029/2005JC003321.

Wahba, G., 1990: Spline Models for Observational Data. SIAM, $169 \mathrm{pp}$.

Weaver, A., and P. Courtier, 2001: Correlation modelling on the sphere using a generalized diffusion equation. Quart. J. Roy. Meteor. Soc., 127, 1815-1846. 
Copyright of Journal of Atmospheric \& Oceanic Technology is the property of American Meteorological Society and its content may not be copied or emailed to multiple sites or posted to a listserv without the copyright holder's express written permission. However, users may print, download, or email articles for individual use. 